\title{
Effects of gamma rays on rat testis tissue according to the morphological parameters and immunohistochemistry: radioprotective role of silymarin
}

\author{
Mohsen Marzban ${ }^{1}$, Maryam Anjamshoa ${ }^{2}$, Parnia Jafari ${ }^{3}$, Hossien Masoumi $^{4}$, Reza Ahadi ${ }^{5}$, Daryoush Fatehi ${ }^{6}$
}

${ }^{1}$ Ph.D. Student of Neuroscience, Faculty of Advanced Medical Technologies, Tehran University of Medical Sciences, Tehran, Iran

${ }^{2}$ Ph.D., Assistant Professor of Anatomical Sciences, Faculty of Medicine, Shahrekord University of Medical Sciences, Shahrekord, Iran

${ }^{3}$ M.D., Researcher in Cellular Oncology, Faculty of Medicine, Shahrekord University of Medical Sciences, Shahrekord, Iran

${ }^{4}$ Ph.D., Assistant Professor of Medical Physics, Faculty of Medicine, Shahrekord University of Medical Sciences, Shahrekord, Iran

${ }^{5} \mathrm{Ph} . \mathrm{D}$. of Neuroscience, Faculty of Paramedicine, Iran University of Medical Sciences, Tehran, Iran

${ }^{6}$ Ph.D., Associate Professor of Medical Physics, Faculty of Medicine, Shahrekord University of Medical Sciences, Shahrekord, Iran

\section{Type of article: Original}

\begin{abstract}
Objective: To determine the radioprotective effects of Silymarin in adult male Sprague-Dawley rats irradiated with $\gamma$-rays.

Methods: The present experimental study was performed in Tehran University of Medical Sciences, Tehran, Iran from December 2009 to March 2010. The study was performed on 40 rats, which were randomly and equally divided into four groups: 1) control group: neither received Silymarin nor irradiated with $\gamma$-rays; 2$) \gamma$-irradiation group: testis region exposed to $2 \mathrm{~Gy}$ of $\gamma$-rays; 3) Silymarin \& $\gamma$-irradiation: rats received $100 \mathrm{mg} / \mathrm{kg}$ of Silymarin $24 \mathrm{hrs}$ before exposure to $2 \mathrm{~Gy}$ of $\gamma$-rays; 4) Silymarin \& $\gamma$-irradiation: rats received $200 \mathrm{mg} / \mathrm{kg}$ of Silymarin $24 \mathrm{hrs}$ before exposure to $2 \mathrm{~Gy}$ of $\gamma$-rays. After animal experiments and preparing the tissue sections, different histological and histomorphological parameters of seminiferous tubules and the biological characteristics of Leydig cells were evaluated applying quantitative assessment, Johnson scoring, and Leydig cell apoptosis assay by TUNEL method. The data were analyzed applying ANOVA and Tukey's post hoc test, using SPSS software (V.19).

Results: Irradiation of $2 \mathrm{~Gy} \gamma$-rays to the testis of the rats significantly affected the frequency of spermatogonia, primary spermatocyte, round spermatid, spermatozoa, seminiferous tube and lumen diameters, thickness of the epithelium, Leydig cell nuclear diameter and volume, epithelium height, and apoptotic cells $(p<0.05)$. However, administration of Silymarin improved the mentioned parameters specifically in $200 \mathrm{mg} / \mathrm{kg}$ of dosage.

Conclusion: Silymarin could act as a potent radioprotector and it can be used in modulation as well as improvement to radiation therapy to prevent male reproductive function, specifically seminiferous tubules in an animal model; however, its molecular mechanism is still not clear and needs more molecular researches.

Keywords: Spermatogenesis, Radiotherapy, Silymarin, Radioprotector, Antioxidant
\end{abstract}

\section{Introduction}

Prostate cancer is the second leading cancer and the fifth leading cause of death in men worldwide. In 2012, 1.1 million men were diagnosed with prostate cancer, of which 307,000 died (1). This cancer is the most common among men in 84 countries, most of which are developed (1). In addition, the incidence of prostate cancer is

\section{Corresponding author:}

Daryoush Fatehi, Department of Medical Physics, Faculty of Medicine, Shahrekord University of Medical Sciences, Shahrekord, Iran. Tel: + 983833335652. Email: d.fatehi@gmail.com. Fax: +983813334911.

Received: September 27, 2016, Accepted: January 15, 2017, Published: June 2017

iThenticate screening: January 07, 2017, English editing: March 01, 2017, Quality control: April 26, 2017

(C) 2017 The Authors. This is an open access article under the terms of the Creative Commons Attribution-NonCommercialNoDerivs License, which permits use and distribution in any medium, provided the original work is properly cited, the use is non-commercial and no modifications or adaptations are made. 
increasing in developing countries (2). By means of prostate-specific antigen test, diagnosis of prostate cancer increased incredibly during the $80 \mathrm{~s}$ and $90 \mathrm{~s}$ (2). Prostate cancer has been reported to be the cause of death in $30 \%$ $70 \%$ of men aged over 60 years who died because of unrelated causes (1). The treatment of this cancer is typically performed in the light of different factors such as tumor growth rate, general health, as well as the benefits and side effects of the treatment. The current prostate cancer treatments are radiotherapy, hormonotherapy, chemotherapy, and cryotherapy (3). Radiotherapy is the most important and common method to treat cancer. Mostly radiotherapy is applied through two procedures: external beam radiation therapy (teletherapy), and internal radiation therapy (brachytherapy) (4). In prostate cancer, both of the methods can be prescribed depending on the general health conditions of the patient, planning, and the physician's opinion. Radiotherapy is aimed at removing tumor cells and preserving surrounding normal cells in the treatment field. However, some parts of organs or tissues might receive notable dosage, which is revealed as either acute or chronic side effects (5). Using protecting medications or radioprotectors could reduce the side effects of radiotherapy. These medications in fact, reduce indirect effects of the radiation (6). Radioprotectors decrease stable hydroxyl radicals in the exposed area, by absorbing reactive oxygen species (ROS) $(6,7)$. Many studies have been performed to determine the effects of such compounds. Some of the studies have confirmed the protective effects of the compounds; while, others have reported unclear or no effects for them (7). Milk thistle (Silybum marianum) was first discovered as a material with antitumor effects, and is being used to treat immune system dysfunctions and alcoholic liver diseases in clinics (8). Milk thistle contains different antioxidants such as Silymarin, which is a potent cell protective agent that reduces oxidative stress and protects the cells against apoptosis. It contains six flavonolignans consisting of $40 \%-50 \%$ silibinin, responsible for most biological activities $(8,9)$. Based on some studies, Silymarin has a radioprotective effect against the radiation induced hepatotoxicity in rats (10). Adhikari M. et al. showed that specific doses of $25 \mu \mathrm{g} / \mathrm{ml}$ Silymarin can ameliorate the deleterious effects of $\gamma$-radiation on human embryonic kidney cells in-vitro (11). Deep G. and Agarwal R. showed that Silymarin, with targeting signaling molecules, could adjust the process of epithelial mesenchymal transition, protease activation, adhesion, and prevention of metastasis (9). Mauduit C. et al. found that by 2-4 Gy of ionizing radiation to adult rats' testes, the apoptotic cells increased after 24 hours and reached to their maximum number after 48 hours (12). Apoptotic cells were observed 72 hours after 2 Gy of radiation, and increased as the dosage increased from 1 Gy to 4 Gy. Growth factor in sensitive testis cells, drops after ionizing radiation (12). Mydlo JH. et al. examined the effects of brachytherapy and external beam radiation therapy on prostate cancer treatment, sperm quality, and fertility in young men. Radiotherapy reduces spermatogenesis by discharging Leydig cells and reduction of testosterone level. It also has direct effects on spermatogenesis with mitosis and abnormal cell division (13). Otala $\mathrm{M}$. et al. used sphingosine-1-phosphate (S1P) to protect sperm against ionizing radiation to rat's testes. They injected S1P to rat's testes 1-2 hours before radiation. Their results showed that use of S1P (16\%-47\%) decreased germ cells' death and protected them (14). Khan S. et al. used melatonin in order to protect mice testes against $\gamma$-irradiation induced damage. Their results showed that melatonin, as a physiological radioprotector, ameliorated the radiation induced damages of epididymal sperm abnormalities such as motility and viability (15). Koruji et al. examined the morphologic changes in adult rat's testes after radiating 14 Gy of $\gamma$-rays. Their results showed that radiation could cause temporary azoospermia potentially reversible after eight weeks (16). Considering the role of radiotherapy in treating different tumors, its induced effects on normal tissues, and the importance of radioprotective substances to reduce the side effects of this method on the region surrounding the tumor, the purpose of this study was to evaluate and determine radioprotective effects of Silymarin on Johnson scoring, histomorphological parameters of seminiferous tubules and biological characteristics of Leydig cells in SpragueDawley male rats irradiated by the same fraction dose of $\gamma$ - rays in prostate cancer radiotherapy.

\section{Material and Methods \\ 2.1. Animals and ethic}

This experimental study was performed in Tehran University of Medical Sciences, Tehran, Iran from December 2009 to March 2010. Forty 9-week-old Sprague-Dawley male rats, weighing 250-300 $\pm 0.2 \mathrm{~g}$, were purchased from the Pasteur Institute (Tehran, Iran). Animals were allowed to have easy access to food and water and were exposed to control conditions of constant illumination (12:12 hours, light from 8 to 20$)$, temperature $\left(23 \pm 2^{\circ} \mathrm{C}\right)$ and humidity (55-65\%); all animals were maintained in cages for one week prior to the start of experiments for acclimatization, and they were eligible to be part of the experiments when they had passed the two consecutive sex cycles. All procedures were carried out in accordance with the "Guide for the Care and Use of Laboratory Animals" prepared by the National Academy of Sciences, and published by the National Institutes of Health (NIH publication 86-23 revised 1985), and also the guidelines of Tehran University of Medical Sciences (Tehran, Iran). 


\subsection{Gamma irradiation}

Gamma irradiation was performed applying a Cobalt-60 source (Theratron 780C, Atomic Energy of Canada Limited, Kanata, ON, Canada). The characteristics of the source were: half-life: 5.27 years, dose rate: 0.5 Gymin-1, medium energy: $1.25 \mathrm{MeV}$. The source had a diameter of $20 \mathrm{~mm}$. The SSD (source to skin distance) was $80 \mathrm{~cm}$ and the CID (collimator to isocenter distance) was $30 \mathrm{~cm}$. Rats were placed in a ventilated Plexiglas cage and irradiated in groups of four, simultaneously, at room temperature of $23 \pm 2{ }^{\circ} \mathrm{C}$. The prostate region (pelvic area and reproductive system) of the rats was irradiated with a total dose of 2 Gy $\gamma$-rays.

\subsection{Grouping and Silymarin administration}

The rats were grouped in 4 groups of 10 . The first group, the control group, were neither treated with Silymarin nor irradiated by $\gamma$-rays; but received intraperitoneal injection of $10 \mathrm{ml} / \mathrm{kg}$ normal saline. The second group were only irradiated with 2 Gy of $\gamma$-rays. The third group was initially treated with $100 \mathrm{mg} / \mathrm{kg}$ of Silymarin (Sigma-Aldrich, S0292-50G, Italy) and then they were irradiated with 2 Gy of $\gamma$-rays 24 hours. later. Similarly, the fourth group was initially treated with $200 \mathrm{mg} / \mathrm{kg}$ of Silymarin and then they were irradiated with 2 Gy of $\gamma$-rays 24 hours later. The solution of Silymarin was administered directly into the stomach of the rats via oral gavage 24 hours before the $\gamma$ irradiation.

\subsection{Histology}

At completion of the experiments, the rats were deeply anesthetized with chloroform and their testes were removed from abdominal cavity. The testes were fixed with $10 \%$ formaldehyde for $72 \mathrm{hrs}$. For light microscopy, the sections were dehydrated by a successive series of ethanol cleared by xylene and impregnated by melted paraffin. Then, the samples were embedded in paraffin wax. Tissues were sectioned at $5 \mu \mathrm{m}$ and stained with hematoxylin and eosin (H\&E) to observe the structure. Then, the slides were examined at 400x magnification.

\subsection{Leydig cell apoptosis assay by TUNEL}

To detect apoptotic cells, TUNEL staining was performed using an apoptosis kit (Roche, Germany) by TUNEL staining according to the manufacturer's instruction. The samples, in paraffin block, were cut into $5 \mu \mathrm{m}$ sections by a rotary microtome, and then placed on glass slides. The sections were deparaffinized in xylene and placed in a 700w microwave for 10 minutes. Then, the sections were incubated with $3 \% \mathrm{H} 2 \mathrm{O} 2$ in PBS for 10 minutes in the dark. Next, the sections were incubated in the TUNEL reaction mixture that contained terminal deoxynucleotidyl transferase and fluorescein-dUTP for 60 minutes at $37^{\circ} \mathrm{C}$ in a humidified atmosphere. Then, the samples were rinsed with PBS three times, the sections were visualized by a converter-POD (Roche Applied Science, Mannheim, Germany) for 30 minutes at $37^{\circ} \mathrm{C}$ in a humidified atmosphere in the dark, and then rinsed with PBS, followed by the addition of 50-100 $\mu 1$ diaminobenzidine (DAB, Cat No: D8001, Sigma-Aldrich) substrate and an additional PBS rinse. The slides were examined by a pathologist in a blinded manner. Only circular tubular cross-sections cut in boldface were studied. The quantitative evaluation of apoptotic index was performed by the manual counting of the mean number of TUNEL-positive cells per interstitial space per group (four sections per rat and 25 interstitial spaces per section). The apoptotic cells in the 100 tubules were recorded using light microscopy at 400x magnification.

\subsection{Quantitative assessment of seminiferous tubules and Johnson scoring}

An ocular grid was used to measure the parameters of the seminiferous tubules. Twenty tubular circular or nearly circular profiles were randomly selected from each animal. Then, two perpendicular diameters of each cross-section of the seminiferous tubules were measured and the mean value was calculated at $400 x$ magnification. In addition, the diameters of each lumen of the somniferous tubules and epithelium height were measured. In this study the spermatogenesis of control and SCI groups were evaluated according to Johnson Score (Table 1).

\subsection{Calculating the volume of Leydig cells and germ cell count}

Using a linear grid and light microscope (Olympus BX51, Germany) at 1000x magnification, the volumes of the nuclei of the Leydig cells were calculated. Thus, per each animal, 20 cells were studied. The number of spermatogonia, primary spermatocytes, round spermatids, and spermatozoa were counted. The location and morphology of the cells within the seminiferous tubules were used to identify them. Each parameter was estimated by the examination of 20 histological sections of each seminiferous tubule.

\subsection{Statistical analysis}

The ANOVA test was applied to compare the averages of the values within each group of Silymarin or $\gamma$ irradiation. Additionally, Tukey's post hoc test was used to compare the four groups. The p-values are two-sided at a 
significance level of $\leq 0.05$. SPSS software (Version 19, SPSS Inc., Chicago, Illinois, USA) was used for the statistical analysis.

Table 1. The calculation criteria of fertility and the duration of spermatogenesis using Johnson scoring

\begin{tabular}{|l|l|}
\hline Seminiferous tubules features & Score \\
\hline $\begin{array}{l}\text { Full spermatogenesis, a large number of sperm heads are located on the sidelines of a round and regular } \\
\text { lumen. }\end{array}$ & 10 \\
\hline Although there are a large number of sperms, no round and regular lumen can be seen. & 9 \\
\hline Sperm count is very low. & 8 \\
\hline There is no sperm, however, a large number of round spermatids are visible & 7 \\
\hline A few round spermatids can be seen & 6 \\
\hline There is no sperm or round spermatid. There is a large number of primary spermatocytes. & 5 \\
\hline Very few primary spermatocytes are seen. & 4 \\
\hline There are no primary spermatocytes. Only spermatogonia is observed & 3 \\
\hline There are no germ cells, only Sertoli cells can be seen. & 2 \\
\hline Neither germ cells nor Sertoli cells are observed and the tubules are atrophic. & 1 \\
\hline
\end{tabular}

\section{Results}

Details of different sperm parameters evaluated in this study are summarized in Tables 2 and 3. Moreover, status of spermatogenesis, histological characteristics of testis, and immunohistochemistry of seminiferous tubules are illustrated in Figures 1 and 2.
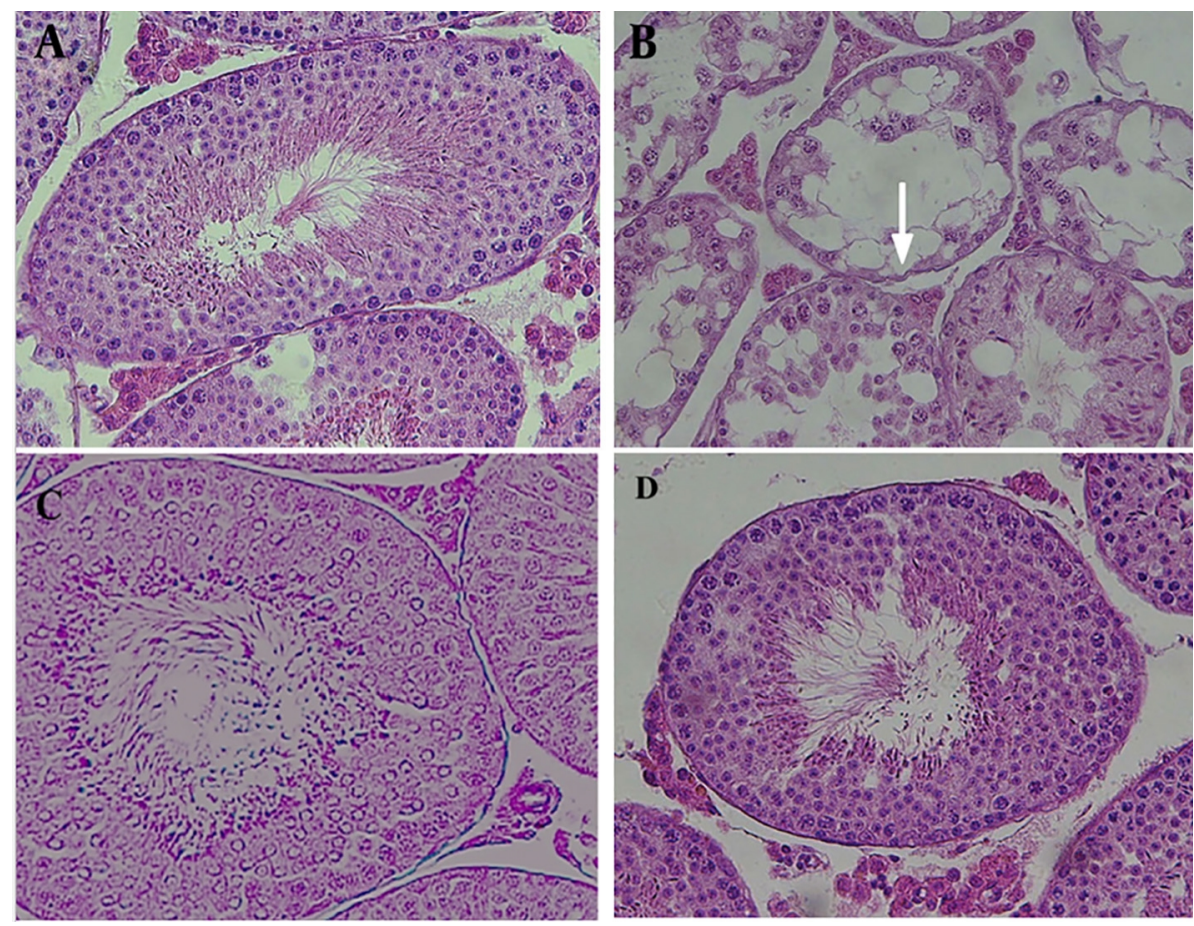

Figure 1. Comparison of the effects of $2 \mathrm{~Gy} \gamma$-irradiation on the testis tissue of Sprague-Dawley rat in the absence and presence of Silymarin in comparison with the control group. A) Control group, B) $\gamma$-irradiation alone group, C) Silymarin (100 mg/kg) \& $\gamma$-rays group and D) Silymarin $(200 \mathrm{mg} / \mathrm{kg}) \& \gamma$-rays group. 

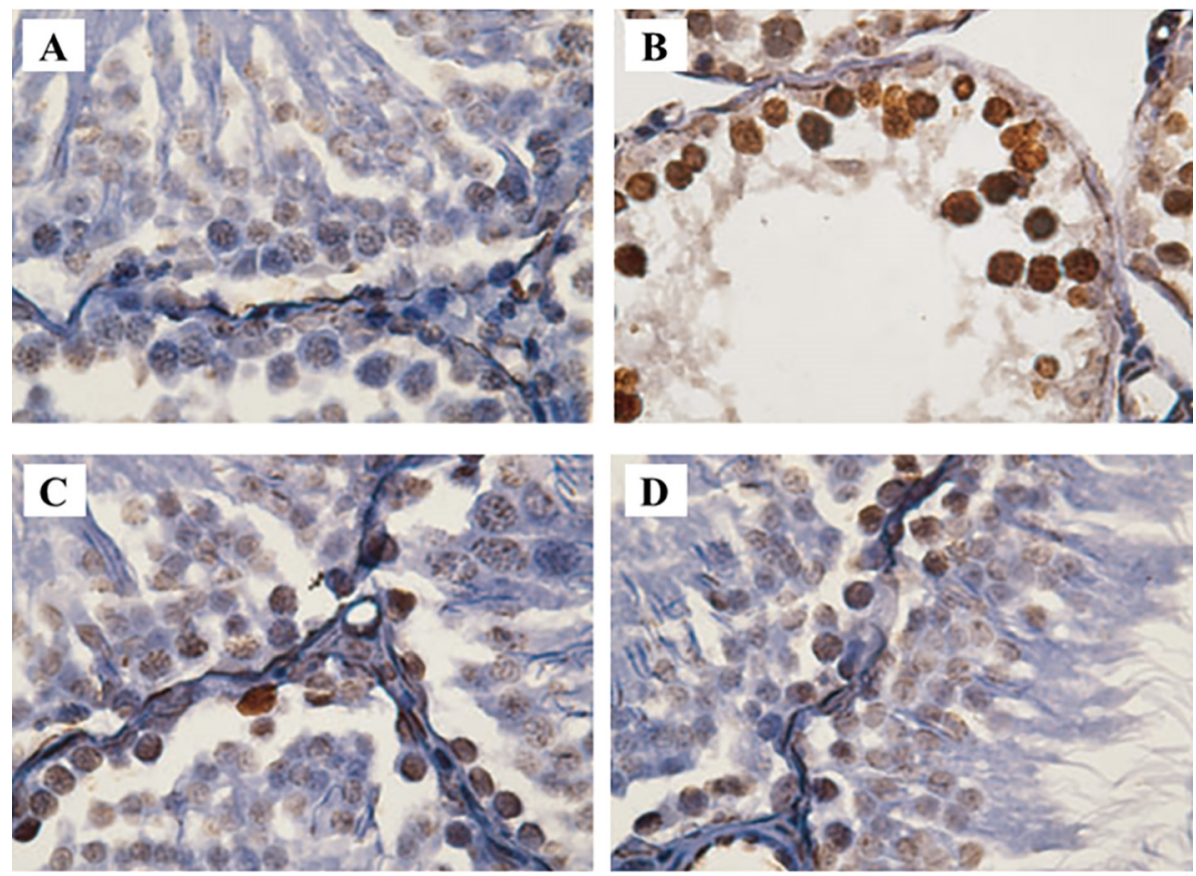

Figure 2. Illustrations of immunohistochemical staining of spermatogonial and primary spermatocyte cells investigated in the present study, comparing the effects of 2 Gy $\gamma$-irradiation on the testis tissue of the SpragueDawley rat in the absence and presence of Silymarin. A) Control group, B) $\gamma$-irradiation alone group, C) Silymarin $(100 \mathrm{mg} / \mathrm{kg}) \& \gamma$-rays group and D) Silymarin $(200 \mathrm{mg} / \mathrm{kg}) \& \gamma$-rays group.

Table 2. The mean $\pm \mathrm{SD}^{*}$ values of different histological parameters of seminiferous tubules as found in the present study.

\begin{tabular}{|l|l|l|l|l|}
\hline Group & $1:$ Control & $\begin{array}{l}2: \gamma \text {-irradiation } \\
\text { alone }\end{array}$ & $\begin{array}{l}\text { 3:Silymarin }(100 \\
\mathrm{mg} / \mathrm{kg}) \& \gamma \text {-rays }\end{array}$ & $\begin{array}{l}4: S i l y m a r i n ~ \\
\mathrm{mg} / \mathrm{kg}) \& \gamma \text {-rays }\end{array}$ \\
\hline Johnson classification & $9.67 \pm 0.26$ & $3.42 \pm 0.42^{\mathrm{a}}$ & $6.16 \pm 0.83$ & $7.64 \pm 0.67^{\mathrm{b}}$ \\
\hline Number of spermatogonia & $3.92 \pm 0.22$ & $1.24 \pm 0.04^{\mathrm{a}}$ & $.02 \pm 1.02$ & $3.27 \pm 0.35^{\mathrm{b}}$ \\
\hline Number of primary spermatocyte & $65.8 \pm 0.24$ & $34.72 \pm 1.03^{\mathrm{a}}$ & $43.11 \pm 1.6$ & $61.73 \pm 0.41^{\mathrm{b}}$ \\
\hline Number of round spermatid & $124.03 \pm 0.94$ & $70.23 \pm 0.82^{\mathrm{a}}$ & $83.79 \pm 0.63$ & $107.5 \pm 1.47^{\mathrm{b}, \mathrm{c}}$ \\
\hline Number of spermatozoa & $128.14 \pm 0.53$ & $86.53 \pm 0.38$ & $92.07 \pm 0.86$ & $119.6 \pm 1.94^{\mathrm{c}}$ \\
\hline
\end{tabular}

a: significant difference compared to the control group (the group $1(\mathrm{p}<0.05)$; b: significant difference compared to the group $2(\mathrm{p}<0.05)$; $\mathrm{c}$ : significant difference compared to the group $3(\mathrm{p}<0.05)$; *SD: standard deviation

Table 3. The mean $\pm \mathrm{SD}^{*}$ values of different histomorphological parameters of seminiferous tubules and the biological characteristics of Leydig cells as found in the present study.

\begin{tabular}{|l|l|l|l|l|}
\hline Group & $1:$ Control & $\begin{array}{l}2: \gamma \text {-irradiation } \\
\text { alone }\end{array}$ & $\begin{array}{l}\text { 3:Silymarin }(100 \\
\mathrm{mg} / \mathrm{kg}) \& \gamma \text {-rays }\end{array}$ & $\begin{array}{l}4: \text { Silymarin }(200 \\
\mathrm{mg} / \mathrm{kg}) \& \gamma \text {-rays }\end{array}$ \\
\hline Tube diameter $(\mu \mathrm{m})$ & $153.27 \pm 0.75$ & $73.09 \pm 1.56^{\mathrm{a}}$ & $104.92 \pm 3.64$ & $126.53 \pm 2.44^{\mathrm{b}, \mathrm{c}}$ \\
\hline Lumen diameter $(\mu \mathrm{m})$ & $78.63 \pm 1.06$ & $33.72 \pm 4.16^{\mathrm{a}}$ & $58.49 \pm 0.04$ & $69.028 \pm 0.62^{\mathrm{b}}$ \\
\hline Thickness of epithelium $(\mu \mathrm{m})$ & $63.96 \pm 0.52$ & $29.18 \pm 2.24^{\mathrm{a}}$ & $42.74 \pm 2.61$ & $47.88 \pm 0.6$ \\
\hline Leydig cell nuclear diameter $(\mu \mathrm{m})$ & $6.39 \pm 0.75$ & $4.07 \pm 0.42$ & $5.84 \pm 0.07$ & $6.23 \pm 1.03$ \\
\hline Leydig cell nuclear volume $(\mu \mathrm{m} 3)$ & $108.72 \pm 0.5$ & $61.83 \pm 1.49^{\mathrm{a}}$ & $83.45 \pm 0.62$ & $101.4 \pm 2.27^{\mathrm{b}, \mathrm{c}}$ \\
\hline $\begin{array}{l}\text { Height of seminiferous epithelium } \\
(\mu \mathrm{m})\end{array}$ & $67.12 \pm 0.83$ & $41.12 \pm 0.64^{\mathrm{a}}$ & $52.11 \pm 1.84$ & $63.07 \pm 0.62^{\mathrm{b}}$ \\
\hline
\end{tabular}

a: significant difference compared to the control group (the group $1, p<0.05)$; b: significant difference compared to the group $2(\mathrm{p}<0.05)$; $\mathrm{c}$ : significant difference compared to the group $3(\mathrm{p}<0.05)$; *SD: standard deviation

\subsection{Histology}

The optical photomicrographs of the cross-sections for the control group indicated the integrity of the tubules and interstitial tissue. The seminiferous tubules were observed completely and clearly. All sperm cell lines were seen 
and the basal membrane of the seminiferous tubules as well as the spermatogonia lines on it could be observed. The Leydig cells in the interstitial tissue were observed with acidophilic cytoplasm. Furthermore, according to Johnson scoring and histological illustration spermatogenesis was visible (96.7\%). Microscopic images showed some unusual spaces and dilations associated with lymphocytes and plasma in group 2, which indicated inflammation in the interstitial tissue. Moreover, in this group, vacuoles were abundantly visible in the interstitial tissue and spermatogonia; while a significant reduction was found for the primary spermatocytes (Figure 1-A). The seminiferous tubules were degenerated, and a decreasing was seen in the diameter of the seminiferous tubules. The seminiferous tubules were disordered and the basement membrane was folded. Epithelium was atrophic, and severe vacuolization could be observed with spermatogonia cells on the basement membrane; however, spermatogenesis could not be observed in all sections. Primary spermatocytes arrested in pachytene stage. More than one third of the spermatogenesis $(38.6 \%)$ could be seen. The Leydig cells in the interstitial tissue had pyknotic nuclei, and were widely distributed. Furthermore, destructive cells with certain features, such as declined cytoplasmic acidophilus and increased nuclear staining, were considerable (Figure 1-B). Microscopic image evaluation of the slices of the $3^{\text {rd }}$ group illustrated that although rate of spermatogenesis was improved (59.2\%); we found a slight reduction in the edema and inflammation of the tissue, as well as mild regenerative effects in the seminiferous tubules. The Leydig cells in the interstitial tissue increased partly with normal appearance, and their cytoplasm was almost acidophilic, which indicates a slight return of necrotic effects at the cellular level. More than half of the sperm cells were seen; however, a reduction in the tubular diameter and thickness, a disruption in germinal epithelium, and an increase in the interstitial space could still be seen (Figure 1-C). In group 4, microscopic images demonstrated that the seminiferous tubules could be clearly seen with all sperm cell lines. The basal membrane of the seminiferous tubules could be seen with spermatogonia lines. The Leydig cells observed in the interstitial tissue, had a completely acidophilic cytoplasm. Spermatogenesis was improved (78.3\%) and the cells had a distinguishable order. Some minor effects of vacuolization were seen; although, edema was still visible in the interstitial tissue (Figure 1-D).

\subsection{Quantitative assessment of seminiferous tubules and Johnson scoring}

The average values of different histological parameters of seminiferous tubules are presented in table 2. As Table 2 shows, Johnson score was significantly decreased in the $2^{\text {nd }}$ group ( $\gamma$-irradiation alone) in comparison to the control group $(\mathrm{p}=0.0097)$. In contrast, comparing the $3^{\text {rd }}$ group [Silymarin $(100 \mathrm{mg} / \mathrm{kg}) \& \gamma$-rays] with the $2^{\text {nd }}$ group, a remarkable, but not significant, increase was found for the Johnson score. Also, a significant increase was seen when we compared the $4^{\text {th }}$ group [Silymarin $(200 \mathrm{mg} / \mathrm{kg}) \& \gamma$-rays] with the $2^{\text {nd }}$ group $(\mathrm{p}=0.014)$. Respecting the Johnson score, we found a remarkable, but not significant, increase comparing the $4^{\text {th }}$ group with the $3^{\text {rd }}$ group. Similar results were found for the other parameters, i.e. average numbers of spermatogonia, primary spermatocyte, round spermatid, and spermatozoa (Table 2). The average values of different histomorphological parameters of seminiferous tubules and the biological characteristics of Leydig cells are shown in Table 3. As Table 3 demonstrates, tube diameter was significantly decreased in the $2^{\text {nd }}$ group when we compared it with the control group ( $\mathrm{p}=0.0078$ ). Comparing the $3^{\text {rd }}$ group with the $2^{\text {nd }}$ group a non-significant increase was found for the tube diameter; however, a significant increase was seen when we compared the $4^{\text {th }}$ group with the $2^{\text {nd }}$ group $(\mathrm{p}=0.0094)$. Regarding the tube diameter, a significant difference was seen between the $4^{\text {th }}$ and the $3^{\text {rd }}$ groups $(\mathrm{p}=0.016)$. Similar results were observed for other parameters of seminiferous tubules, i.e. lumen diameter, primary spermatocyte, and thickness of the epithelium. Additionally, similar results were found for nuclear diameter and nuclear volume of the Leydig cells. Furthermore, similar results were seen for the height of the seminiferous epithelium (Table 3).

\subsection{Leydig cell apoptosis assay by TUNEL}

Immunohistochemistry illustrations show the average of the apoptotic cells which turned brown in group 2 was significantly higher than those of the control group ( $8.24 \pm 0.53)$ vs. $1.03 \pm 0.65)$; respectively $(\mathrm{p}=0.012)$. Also, quantitative analysis of TUNEL assay showed apoptotic index in spermatocyte I cells were significantly increasing in group II. The frequency of apoptotic cells per 100 tubules in groups 3 and 4 was $5.17 \pm 1.92$ and 3.96 \pm 1.04 , respectively; which represented a significant decrease in the number of damaged cells in group 4 compared to group $2(\mathrm{p}=0.023)$. This also showed that animals which received $200 \mathrm{mg} / \mathrm{kg}$ of Silymarin had some improvements in support reproductive cells and their testes (Figure 2).

\section{Discussion}

The results of the present study demonstrated that irradiation of 2 Gy $\gamma$-rays to the testis of the Sprague-Dawley rat, significantly reduced different histological parameters of seminiferous tubules (i.e. frequency of spermatogonia, primary spermatocyte, round spermatid, spermatozoa. Furthermore, our results showed that $\gamma$-irradiation could destruct and induce roughly stable damages of different histomorphological parameters of seminiferous tubules and 
the biological characteristics of Leydig cells. However, the mentioned parameters were modulated and or significantly improved when we administered $100 \mathrm{mg} / \mathrm{kg}$ (and or $200 \mathrm{mg} / \mathrm{kg}$ ) of Silymarin to the Sprague-Dawley rat, 24 hours before 2 Gy $\gamma$-irradiation. Apoptosis is an event that occurs in association with extracellular molecules as well as intracellular messages, and protects the cells against spontaneous cell suicide. This process occurs in response to cytotoxic agents through spermatogenesis, making a balance between healthy and dead germ cells (17). Apoptosis in testis tissue usually occurs in A2, A3 and A4 stages of spermatogonia; however, apoptosis rarely occurs in the spermatocytes of the stages 1 and 2 as well as spermatids; unless specific environmental and chemical factors induce this process (18). Testis tissue is highly active with poor cell renewal system and antioxidant defense, which can be an easy target for the induction of radiation through the production of the free radicals that cause indirect and reversible damages (19). Oxidative stress in the testes can disrupt the estrogen capacity of the Leydig cells. The capacity of germinal cells (germ) is different from normal spermatozoa, leading to impaired spermatogenesis and infertility through the oxidation of proteins, lipids, and germ cells' DNA (20). DNA bases are sensitive to oxidative stress, and the peroxidation of structures can cause basal modifications, DNA chain breaks, and the exchange of chromatin components. Free radicals produced by ionizing radiation, are the cause of diseases and cell damage, especially widespread infections (21). Radiation-induced DNA damages are a very important cause of deleterious effects on reproductive cells which may accelerate the process of chromosomal aberrations, gene mutations, apoptosis of germ cells, and sustainable development of testes atrophy; which ultimately leads to longterm azoospermia and infertility $(15,19)$. Radiation not only interferes with the cellular antioxidant defense system, it also contributes to alteration of mitochondrial electron transport chain which is one of its main cellular targets, and destroys cell divisions in the germ cells, and ultimately causes pyknosis and karyolysis $(19,21,22)$. Radiation also increases germ cell death in testes by activating caspase 3, which are the main mediators of apoptotic pathway (or play essential roles in apoptosis), and thereby reduces the thickness of germinal cell epithelium and the atrophy of the testes tissue $(14,22)$. Silymarin has certain anti-inflammatory effects causing reduction in the inflammation and edema in the testes tissues of groups 3 and 4. This substance probably inhibits the migration of leukocytes and increases neutrophil infiltration in the area of inflammation, which plays a key role in the anti-inflammatory action (23). Silymarin also stabilizes the membrane structures, and inhibits the release of inflammatory mediators such as cyclooxygenase and lipoxygenase. Silymarin decreases the expression of the growth factor TGF- $\beta 1$, prevents the activation of NF-kB and cell proliferation-related kinases such as TNF- $\alpha$, and decreases cytotoxicity to avoid apoptosis $(11,24)$. Due to having antioxidant property, Silymarin is able to scavenge free radical and ROS. It prevents the progression of cell damage and apoptosis by inhibiting lipid peroxidation (24). The results obtained in the present study, demonstrated certain changes in spermatogonia, spermatocytes, spermatid, and the number of spermatozoa 24 hours after $\gamma$-irradiation. This study also showed direct effects of $\gamma$-radiation, a sharp decline in all the categories of Johnson's scoring, number of spermatogonia, spermatocytes, spermatid cycle, and spermatozoa, which confirms the results obtained by Mauduit C. et al \& Koruji M. et al. (12, 16). Similarly, Chuai Y. et al reported that the highest reduction $(72.9 \%)$ in the number of spermatogonia occurred after one-week irradiation with 2 Gy (25), while the corresponding reductions in our study were derived as $61.4 \%, 40.8 \%$, and $21.7 \%$ in the groups of $\gamma$-irradiation alone (II), Silymarin (100 mg/kg) (III), and (200 mg/kg) (IV), respectively. They concluded that their results represent radioprotective property of Silymarin. Additionally, due to nontoxicity and noninvasive effects of Silymarin, its concentration can increase its radioprotective effects in external radiation treatment (26). In this study, the optimal concentration of Silymarin to protect germ cells against ionizing radiation was not investigated; however, we suggest performing it in the future. The results obtained in our study for these parameters in groups 3 and 4 confirm the radioprotective effect of Silymarin on the germ cells. Antioxidant molecules such as Silymarin can modulate the oxidative status of cell environment via increase in cell glutathione, and prevents lipid peroxidation, resulting in improvement of the antioxidant defense system (24). Silymarin has been able to decrease the development of neurons in cerebral ischemia as well as improvement of functional recovery, as the results show that the neuroprotective potential of Silymarin is due to its antioxidant and antiapoptotic properties (27). As recommendations, the germ cell and sperm production in the mammalian testis depends on normal Leydig cell function and its biochemical interactions with germ cells which is a pivotal mechanism in spermatogenesis process. Epithelium of seminiferous tubules in mammals is a very complex but well-organized cell population that is made up of germ cells with different levels of sensitivity to radiation. Leydig cells and Sertoli cells, although closely related to germ cells, have been reported to be relatively sensitive to radiation. Radiation via ROS production is capable of disrupting the steroidogenic capacity of Leydig cells, and also decrease in sperm count, thus resulting in germ cell apoptosis, disruption of spermatogenesis and fertility capacitation. Silymarin target mitochondrial ROS, and scavenge the free radicals during the radiation exposure as part of a strategy to mitigate cellular stress and apoptosis. In conclusion, biological protection of male fertility against radiation hazard, is a major concern about any other harmful effects of radiation that may be transmitted from one generation to another. Understanding the 
radiation sensitivity of germ cells, and strengthening them, paves the way for the development of radiation moderators as well as radioprotective agents that can be effectively used for high-sensitivity protection of germ cells against the harmful effects of ionizing radiation. In this study, there were limitations which prolonged the time of the study or stopped some of the stages including 1) lack of access to testing kits TUNEL and late arrival to the site of the project 2) lack of advanced machines for precise image analysis and lack of software for cell counts or cell morphometry 3) the unavailability of irradiation devices for small animals and preclinical equipment for exactness and similarity of radiation similar to human radiation devices.

\section{Conclusions}

In this study, we investigated the radioprotective effect of Silymarin on the histological and histomorphological parameters of seminiferous tubules as well as the biological characteristics of Leydig cells of adult male SpragueDawley rats irradiated with 2 Gy $\gamma$-rays. Our results showed that Silymarin can improve the histological and histomorphological parameters of seminiferous tubules such as frequency of spermatogonia, primary spermatocyte, round spermatid, spermatozoa, tube diameter, lumen diameter, thickness of epithelium, Leydig cell nuclear diameter, Leydig cell nuclear volume, and height of seminiferous epithelium, as well as apoptotic cells. Therefore, one can conclude that Silymarin could act as a potent radioprotector and it can be used adjacent to radiation therapy to prevent male reproductive function, specifically seminiferous tubules in an animal model; however, its molecular mechanism is still not clear, and needs more molecular researches.

\section{Acknowledgments:}

This study was supported by a grant from the Research Institute for Islamic and Complementary Medicine, No. p26/m/t/1088. Also, the authors would like to thank the lab staff of the Cellular and Molecular Research Center in Tehran University of Medical Sciences for their kind cooperation. The authors appreciate Dr. Mehdi Mehdizadeh for his technical assistance.

\section{Conflict of Interest:}

There is no conflict of interest to be declared.

\section{Authors' contributions:}

All authors contributed to this project and article equally. All authors read and approved the final manuscript.

\section{References:}

1) Ferlay J, Soerjomataram I, Dikshit R, Eser S, Mathers $C$, Rebelo M, et al. Cancer incidence and mortality worldwide: sources, methods and major patterns in GLOBOCAN 2012. Int J Cancer. 2015; 136(5): E35986. doi: 10.1002/ijc.29210. PMID: 25220842.

2) Baade PD, Youlden DR, Krnjacki LJ. International epidemiology of prostate cancer: geographical distribution and secular trends. Mol Nutr Food Res. 2009; 53(2): 171-84. doi: 10.1002/mnfr.200700511. PMID: 19101947.

3) Chen FZ, Zhao XK. Prostate cancer: current treatment and prevention strategies. Iran Red Crescent Med J. 2013; 15(4): 279-84. doi: 10.5812/ircmj.6499. PMID: 24082997, PMCID: PMC3785898.

4) Fitzgerald TJ, Wang T, Goel HL, Huang J, Stein G, Lian J, et al. Prostate carcinoma and radiation therapy: therapeutic treatment resistance and strategies for targeted therapeutic intervention. Expert Rev Anticancer Ther. 2008; 8(6): 967-74. doi: 10.1586/14737140.8.6.967. PMID: 18533806, PMCID: PMC2764989.

5) Baskar R, Lee KA, Yeo R, Yeoh KW. Cancer and radiation therapy: current advances and future directions. Int J Med Sci. 2012; 9(3): 193-9. doi: 10.7150/ijms.3635. PMID: 22408567, PMCID: PMC3298009.

6) Rosen EM, Day R, Singh VK. New approaches to radiation protection. Front Oncol. 2015; 4: 381. doi: 10.3389/fonc.2014.00381. PMID: 25653923, PMCID: PMC4299410.

7) Hazra B, Ghosh S, Kumar A, Pandey BN. The prospective role of plant products in radiotherapy of cancer: a current overview. Front Pharmacol. 2012; 2: 94. doi: 10.3389/fphar.2011.00094. PMID: 22291649, PMCID: PMC3253585.

8) Karimi G, Vahabzadeh M, Lari P, Rashedinia M, Moshiri M. "Silymarin", a promising pharmacological agent for treatment of diseases. Iran J Basic Med Sci. 2011; 14(4): 308-17. PMID: 23492971, PMCID: PMC3586829.

9) Deep G, Agarwal R. Antimetastatic efficacy of silibinin: molecular mechanisms and therapeutic potential against cancer. Cancer Metastasis Rev. 2010; 29(3): 447-63. doi: 10.1007/s10555-010-9237-0. PMID: 20714788, PMCID: PMC3928361. 
10) Ramadan LA, Roushdy HM, Abu Senna GM, Amin NE, El-Deshw OA. Radioprotective effect of silymarin against radiation induced hepatotoxicity. Pharmacol Res. 2002; 45(6): 447-54. doi: 10.1006/phrs.2002.0990. PMID: 12162944.

11) Adhikari M, Dhaker A, Adhikari J, Ivanov V, Singh V, Chawla R, et al. In vitro studies on radioprotective efficacy of silymarin against $\gamma$-irradiation. Int $\mathrm{J}$ Radiat Biol. 2013; 89(3): 200-11. doi: 10.3109/09553002.2013.741285. PMID: 23078259.

12) Mauduit C, Siah A, Foch M, Chapet O, Clippe S, Gerard JP, et al. Differential expression of growth factors in irradiated mouse testes. Int J Radiat Oncol Biol Phys. 2001; 50(1): 203-12. doi: 10.1016/S03603016(01)01461-4. PMID: 11316565.

13) Mydlo JH, Lebed B. Does brachytherapy of the prostate affect sperm quality and/or fertility in younger men? Scand J Urol Nephrol. 2004; 38(3): 221-4. doi: 10.1080/00365590410025451. PMID: 15204375.

14) Otala M, Suomalainen L, Pentikäinen MO, Kovanen P, Tenhunen M, Erkkilä K, et al. Protection from radiation-induced male germ cell loss by sphingosine-1-phosphate. Biol Reprod. 2004; 70(3): 759-67. doi: 10.1095/biolreprod.103.021840. PMID: 14613902.

15) Khan S, Adhikari JS, Rizvi MA, Chaudhury NK. Radioprotective potential of melatonin against ${ }^{60}$ Co $\gamma-$ ray-induced testicular injury in male C57BL/6 mice. J Biomed Sci. 2015; 22: 61. doi: 10.1186/s12929-0150156-9. PMID: 26205951, PMCID: PMC4514449.

16) Koruji M, Movahedin M, Mowla SJ, Gourabi H, Arfaee AJ. The morphological changes of adult mouse testes after 60Co gamma-Radiation. Iran Biomed J. 2008; 12(1): 35-42. PMID: 18392093.

17) Shaha C, Tripathi R, Mishra DP. Male germ cell apoptosis: regulation and biology. Philos Trans R Soc Lond B Biol Sci. 2010; 365(1546): 1501-15. doi: 10.1098/rstb.2009.0124. PMID: 20403866, PMCID: PMC2871916.

18) Albuquerque AV, Almeida FR, Weng CC, Shetty G, Meistrich ML, Chiarini-Garcia H. Spermatogonial behavior in rats during radiation-induced arrest and recovery after hormone suppression. Reproduction. 2013; 146(4): 363-76. doi: 10.1530/REP-12-0494. PMID: 23884860.

19) Han Y, Son SJ, Akhalaia M, Platonov A, Son HJ, Lee KH, et al. Modulation of radiation-induced disturbances of antioxidant defense systems by ginsan. Evid Based Complement Alternat Med. 2005; 2(4): 529-36. doi: 10.1093/ecam/neh123. PMID: 16322811, PMCID: PMC1297496.

20) Aitken RJ, Roman SD. Antioxidant systems and oxidative stress in the testes. Oxid Med Cell Longev. 2008; 1(1): 15-24. PMID: 19794904, PMCID: PMC2715191.

21) Szumiel I. Ionizing radiation-induced oxidative stress, epigenetic changes and genomic instability: the pivotal role of mitochondria. Int J Radiat Biol. 2015; 91(1): 1-12. doi: 10.3109/09553002.2014.934929. PMID: 24937368.

22) Azzam EI, Jay-Gerin JP, Pain D. Ionizing radiation-induced metabolic oxidative stress and prolonged cell injury. Cancer Lett. 2012; 327(1-2): 48-60. doi: 10.1016/j.canlet.2011.12.012. PMID: 22182453, PMCID: PMC3980444.

23) Dehmlow C, Erhard J, de Groot H. Inhibition of Kupffer cell functions as an explanation for the hepatoprotective properties of silibinin. Hepatology. 1996; 23(4): 749-54. doi: 10.1053/jhep.1996.v23.pm0008666328. PMID: 8666328.

24) Surai PF. Silymarin as a Natural Antioxidant: An Overview of the Current Evidence and Perspectives. Antioxidants (Basel). 2015; 4(1): 204-47. doi: 10.3390/antiox4010204. PMID: 26785346, PMCID: PMC4665566.

25) Chuai Y, Shen J, Qian L, Wang Y, Huang Y, Gao F, et al. Hydrogen-rich saline protects spermatogenesis and hematopoiesis in irradiated BALB/c mice. Med Sci Monit. 2012; 18(3): BR89-94. PMID: 22367121, PMCID: PMC3560739.

26) Dunnick JK, Singh B, Nyska A, Peckham J, Kissling GE, Sanders JM. Investigating the potential for toxicity from long-term use of the herbal products, goldenseal and milk thistle. Toxicol Pathol. 2011; 39(2): 398-409. doi: 10.1177/0192623310394211. PMID: 21300790.

27) Raza SS, Khan MM, Ashafaq M, Ahmad A, Khuwaja G, Khan A, et al. Silymarin protects neurons from oxidative stress associated damages in focal cerebral ischemia: a behavioral, biochemical and immunohistological study in Wistar rats. J Neurol Sci. 2011; 309(1-2): 45-54. doi: 10.1016/j.jns.2011.07.035. PMID: 21840019. 